IJBPAS, December, Special Issue, 2021, 10(12): 236-245

ISSN: 2277-4998

International Journal of Biology, Pharmacy and Allied Sciences (IJBPAS)

'A Bridgs Betwen Labortory and Qanda'

WwW.iibpas.com

\title{
ROLE OF A POLYHERBAL COMBINATION STREE SANJIVANI IN FEMALE GYNECOLOGICAL DISORDERS
}

\section{SMITA NARAM ${ }^{1}$, DEEPAK MAHAJAN ${ }^{2}$, HEMANG PAREKH ${ }^{3}$ AND NORMA REBELLO ${ }^{4}$}

1: Department of Research and Development, Ayushakti Ayurveda Pvt Ltd, Bhadran nagar cross road, Malad, Mumbai-64

2: Research Head, Ayushakti Ayurveda Pvt Ltd, Bhadran nagar cross road, Malad, Mumbai- 64

3: Medical Head, Ayushakti Ayurveda Hospital, Bhadran nagar cross road, Malad, Mumbai-64

4: Associate Professor in Pharmaceutics, St. John Institute of Pharmacy and Research, St.

John Technical Campus, Vevoor village, Manor Road, Palghar (E)-401404, Dist: Palghar, Maharashtra, India

*Corresponding Author: E Mail: Dr. Deepak Mahajan: drdeepakm@ayushakti.com Received $9^{\text {th }}$ May 2021; Revised $10^{\text {th }}$ July 2021; Accepted $29^{\text {th }}$ Aug. 2021; Available online $15^{\text {th }}$ Dec. 2021 https://doi.org/10.31032/IJBPAS/2021/10.12.1021

\section{ABSTRACT}

Female reproductive health is a crucial aspect to be looked at and the utmost priority for all the women. Polycystic ovarian syndrome, pre-menstrual syndrome, anovulation, menorrhagia, painful menstruation, hormonal imbalance, irregular menstrual cycles, heavy uterine bleeding, endometriosis, leucorrhoea, inflammation, miscarriage, female infertility etc. are the few disorders frequently reported in females. Most of these gynecological problems are neglected in the nascent stage, go untreated and hence may progress to an untreatable stage. Traditional system of medicines such as Ayurveda mentions beneficial effects of herbs in providing relief from female reproductive disorders, maintaining the healthy state as well as restoring the abnormalities. Several herbs such as Vitus agnus castus, Hemidesmus indica, Asparagus racemosus, Saraca indica, Glycerrhiza glabra, Bacopa monneiri etc. have been reported to possess potential benefits to overcome the abnormalities humans. Anti-inflammatory, antioxidant, restoring hormonal balance, aiding oocyte 
maturation etc. Are some of the advantageous actions exhibited by herbs to aid female reproductive health. However those specific herbs and species may not be always available handy and in appropriate composition of the phytoconstituents and are known only to the few vaidya or ayurvedic practitioners. In addition to herbal actives, elemental micronutrients are also useful in treating such disorders. Stree sanjivani thus aimed to provide a holistic care by providing several ayurvedic actives in a single product and catering to multiple disorders.

\section{Keywords: Gynecological problems, female reproductive health, herbs}

\section{INTRODUCTION:}

Women constitute the most important pillars of the family. Their reproductive health is an utmost crucial aspect to be looked at and be maintained to ensure the growth and prosperity in the family. However most often the female reproductive organ abnormalities and disorders are either neglected or goes untreated unless its severe. Polycystic Ovarian Syndrome, Premenstrual syndrome, Irregular Menstruation, Menorrhagia as well an anovulation tops the reproductive health concern majorly. With the revolutionary transformation in the global medical technology as well as enormous awareness among the females today the appropriate diagnosis of such disorders has been made available at the tip of the finger even in the remote regions. With advances in the allopathic system of medicine the cure is also possible however many a times the undesirable effects of the drugs or hormonal treatment led females to opt for the alternative systems.

Indegenoius system of medicine holds promising potential to cure mankind from various ailments. Such traditional systems of medicine of the respective countries are thus treasured with utmost care. Ayuveda, the most ancient and exhaustive Indian medicinal system reports several herbs and their extracts to treat the pathological state as well as restore thephysiological imbalance of the living organisms. Several herbs such as Vitus agnus castus, Asparagus racemosus, Hemidesmus indicus, Saraca indica, etc. As well as ayurvedic dosage forms have been reported to provide significant relief by catering the root cause of the abnormalities rather than a symptomatic one. Taking advantage of the literature based evidences the present article focusses on the selected Ayurvedic herbs in the treatment of Female Reproductive disorders and introduce Stree Sanjivani a unique combination manufactured by Ayushakti Ayurveda Pvt Ltd.

\section{Name of Herbal Combination}

Tablet Stree Sanjivani.

Manufacturer : 
Ayushakti Ayurveda Pvt Ltd pharmacy, Nashik- 422112.

Plot number 78, Stice, Musalgaon, Sinnar,

\begin{tabular}{|c|c|c|c|c|}
\hline \multicolumn{1}{|c|}{ Herbal formulation } & Part used & qty in $\mathbf{g}$ \\
\hline Sr. no. & Sanskrit name & Latin name & Seed & $\mathbf{4 0 0 . 0 0}$ \\
\hline $\mathbf{1}$ & Renuka beej & Vitex agnus castus & Root & $\mathbf{1 0 0 . 0 0}$ \\
\hline $\mathbf{2}$ & Shatavari & Asparagus racemosus & Root & $\mathbf{1 0 0 . 0 0}$ \\
\hline 3 & Sariva & Hemidesmus indicus & Bark & 75.00 \\
\hline 4 & Yashtimadhu & Glycyrrhiza glabra: & Bark & 100.00 \\
\hline 5 & Ashoka & Saraca indica & Whole plant & $\mathbf{2 5 . 0 0}$ \\
\hline 6 & Brahmi & Bacopa monnieri & $\mathbf{5 . 0 0}$ \\
\hline 7 & Yashada bhasma & Zincum & & \\
\hline
\end{tabular}

\section{Vitex agnus castus:}

Fruits of the plant Vitex agnus castus constitutes various phytoconstituents such as iridoids, flavonoids, diterpenoids, essential oils and ketosteroids [1]. Traditionally these fruits have shown significant efficacy in the treatment of female reproductive health, specifically in premenstrual disorders and fertility. The fruit extracts exhibit its beneficial actions by catering to hormonal imbalances mostly commonly in females. Through its action on pituitary gland the fruit extracts aid to increase secretion of luteinizing hormone thereby increasing the production of progesterone. Thus the herb could be useful in the treatment of fibroids, premenstrual syndrome (PMS), mood swings, as well as perimenopausal times of increased estrogen [2]. A randomised, placebo controlled study enrolling 178 women volunteers with a prior history of PMS for three consecutive menstrual cycles revealed a significant advantage of the $V$. agnus castus fruit contents compared to placebo in controlling the premenstrual symptoms such as irritability, mood alteration, anger, headache, bloating and breast fullness [3]. Exemplary benefits of the herb in treating premenstrual syndrome as well as preventing miscarriage in a randomized control trials have also been reported by others $[4,5]$. Additionally $V$. agnus castus is reported to alleviate symptoms associated with latent hyperprolactinemia, i.e lower-than-normal progesterone secretion and normal to mildly elevated prolactin levels. These characteristics are believed to cause a number of menstrual disorders associated with the alteration in the hypothalamic-pituitary axis. Phytoestrogenic activities of $V$. agnus castus extracts were also reported in to treat altered menstrual cycle lengths, and amenorrhea owing to its ability to inhibit prolactin secretion mediated through competitive binding to dopamine D2, opioid receptors as well as capability to enhance melatonin secretion thereby effective in treating menopausal symptoms [6]. Vitex agnus castus, is thus reported to be an adjunct in fertility treatment 
exhibiting exemplary benefits in treating anovulatory cycles, hyperprolactinemia, hypothalamic dysfunction, hypothalamicpituitary-ovarian (HPO) axis modulation, and promoting a regular and normal menstrual cycle.

\section{Hemidesmus indicus:}

Traditional Ayurvedic literature reports the use of roots of the plant Hemidesmus indicus in the treatment of menorrhagia i.e abnormal heavy menstrual bleeding. The symptoms also include pain during menstruation (Dysmenorrhoea), intermenstrual bleeding (Metrorrhagia), prolonged or excessive uterine bleeding (Menometrorrhagia), absence or missed menstruation (Amenorrhea). The roots of H. indicus constitutes essential oils, aromatic methoxybenzaldehydes (vanillin, 3-hydroxy 4-methoxy benzaldehyde or isovanillin), and derivatives (2-hydroxy 4methoxy benzoic acid or HMBA). The marker compound of $H$. indicus, 2-hydroxy 4-methoxy benzaldehyde (MBAlD), accumulated mostly in roots, is also reported with tyrosinase and acetylcholinesterase inhibitory, antiophidian, anti-diabetic, hepatoprotective as well as larvicidal activities [7]. The hydroalcoholic root extract exhibited antileukemia activity through its ability to increase cell apoptosis, necrotic cell fraction, mitochondrial fraction as well as caspase 8 and caspase 3 activity [8].
Immunostimulatory activity was reported by using the root decoction through its ability to increase CD14-derived dendritic cells maturation, caspase 8 activity, cell death, cleaved PARP expression [9]. Ethanolic and methanolic root extract also exhibited neuroprotective activity when studied using Swiss Albino mice and was found to increase the catalepsy duration, brain antioxidant enzyme levels, as well as serotonin, dopamine, 5-HT levels while decreasing the glutamate, acetylcholinesterase and MAO-B enzyme levels. Free-radical mediated oxidative stress and hepatotoxicity was also found to be overcomed by administration of ethanolic root extract. Similarly Crude methanol extract of $H$. indicus root and methanol extract was reported to exhibit prophylactic and therapeutic antiinflammatory activity respectively by inhibiting secretory protein SPI-1 (responsible for invasion and enteritis) and SPI-2 (related in intracellular survival and multiplication) of rat intestine. Antiinflammatory mediated anti-asthmatics effect was also studied and the ethanolic root extract inhibited the clonidine induced catalepsy via antagonizing $\mathrm{H} 1$ receptor or by suppressing mast cell clonidine induced degranulation.

Asparagus racemosus

Asparagus racemosus also known as Shatavari is a well-known Ayurvedic 
remedy indicated for use in female

reproductive problems [1]. Alcoholic extract of shatavari rhizome was found to exert estrogenic effects on the female mammary glands as well as genital organs in adult pregnant female rats [11]. Shatavari exhibits phytoestrogenic properties hence effectively helps to correct the hormonal imbalance and regulate estrous or ovarian cycle in mammals as well as reduces adverse menopausal symptoms [12]. Clinical studies employing Shatavari have been reported to prove beneficial effects in combating premenstrual syndrome (PMS) and dysmenorrhoea by regulating the pituitary gland functions to improve follicular growth, development and ovulation, and importantly reduce PCOS thus suggested to successfully manage PCOS-mediated subfertility. Reactive Oxygen Species (ROS) mediated stress deteriorates the oocyte thereby leading to infertility. Shatavari treatment was found to increase glutathione content and glutathione peroxidase activity in mouse brain while the methanolic extract exhibited increased superoxide dismutase, catalase, and ascorbic acid and decreased lipid peroxidation in rat. Hence antistress and antioxidant property of Shatavari extract could be beneficial in maintaining the appropriate oocyte quality [13]. Steroidal saponin from shatavari are also reported to block oxytocin- induce uterine contractions in rat, rabbit and guinea pig in vivo as well as in situ. Ethyl acetate and acetone extracts of roots of A. racemosus effectively blocked the spontaneous motility of the virgin rat's uterus and inhibited the spasm genic effect of Acetyl chloine, barium chloride and serotonin on the uterus, thus confirming its ability to relieve from dysmenorrhea [14].

\section{Saraca indica:}

Traditional Ayurveda reports Saraca indica or Asoka bark for treating menorrhagia i.e to stop excessive uterine bleeding, uterine disorders and stop menstrual pain $[\mathbf{1 5}, \mathbf{1 6}]$. The bark extract also helps to cure leucorrhoea. These actions are mediated due to the presence of several polyphenolic constituents such as epicatechin, catechin, procyanidin p2, 11-deoxyprocyanidin B, procyanidin, leukocyanidin, etc. Aqueous bark extract exhibited stimulation of the uterine muscle hence provide more frequent contractions [17]. Oxytocic activity of the plant was seen in rat and human isolated uterine preparations. Alcoholic bark extract was found to be effective in Estrogen-primed or gravid uterus as well. ${ }^{18}$ Asoka is a good uterine tonic and keeps the menstruation clear while in pregnancy the extract do not cause abortion. Methanolic and aqueous extract was reported to also exhibit antimicrobial activity. 
Glycyrrhiza glabra:

The chief active phytoconstituent present in the roots of Glycyrrhiza glabra (Liquorice) is a triterpenoid saponin, glycyrrhizin comprising $10 \%$ of the dry weight. It is 50 times sweeter than sucrose and is present as a mixture of potassium, calcium and magnesium salts of glycyrrhizic acid. Additionally several flavonoids of the class flavanones, flavones, flavanonols, chalcones, isoflavans, isoflavenes, isoflavones, and isoflavanones are present in the liquorice roots [19]. The major flavonoids are glycosides of liquiritigenin (4',7-dihydroxyflavanone) and isoliquiritigenin $\left(2^{\prime}, 4,4^{\prime}-\right.$ trihydroxychalcone), such as liquiritin, isoliquiritin, liquiritin apioside, and licuraside [20]. Glycyrrhizin and its aglycone, $18 \beta$-glycyrrhetinic acid possess antitumor activities in breast and ovarian cancer, gastric tumors, and leukemia. Glycyrrhetinic acid and its derivatives were reported to exhibit promising cytotoxicity on human breast cancer cell lines (MCF-7, MDA-MB-231). The ethanolic liquorice extract containing 18 $\beta$-glycyrrhetinic acid mediated estrogenic effect via its agonist activity on MCF-7 breast cancer cells [21]. Similarly the proven anticancer activity inhuman leukaemia was reported by inducing the cell apoptosis through the activation of extrinsic and intrinsic apoptotic pathways. Licochalcone E, the reported isoflavone was found to influence sexual development by impairing the oestrus cycle as well as ovarian, hypothalamus and pituitary gland functions [22]. Glabridin another phytoconstituent present id liquorice root is a common component of herbal remedies used for the treatment of menopausal symptoms, resulting in favorable outcomes similar to those of 17 $\beta$-oestradiol [23]. Isoliquiritigenin also possess a strong oestrogen-like activity and suggested to be cyclized to liquiritigenin, an active flavonoid under physiological conditions. The stimulatory effects of glabrene were found to be similar to those of oestradiol. Interestingly isoliquiritigenin and formononetin was found to stimulate sperm during fertilization [1]. Thus these phytoestrogens exhibit useful therapeutic agents for infertility treatments. The alcoholic extract of $G$. glabra has antiandrogenic effects probably by increasing the testosterone metabolism, down-regulating androgen receptors, or activating oestrogenic receptors. Apart from all the earlier reported gynacological uses Liquorice root extracts also exhibit antiviral activity against herpes simplex and other viruses, thus predicted to exert anti HIV activity and hence sexually transmitted HIV virus could be catered. It acts via its ability to inhibit virus gene expression and replication, decreasing the 
adhesion force and stress and reducing HMGB1 binding to DNA. Glycyrrhizin and $18 \beta$-glycyrrhetinic acid were reported to enhance host cell activities by blocking the degradation of I $\kappa \mathrm{B}$ enzyme involved in the propagation of the cellular response to inflammation, activate $\mathrm{T}$ lymphocyte proliferation, and suppress host cell apoptosis thus inhibiting the adsorption and penetration of the virus in the early steps of the replicative cycle.

\section{Bacopa monnieri:}

Bacopa monnieri extract mainly is reported as brain tonic due to its antistress, antioxidant and cognition facilitator properties [24]. Several Reports reveal antifertility potential of bacopa extracts in male as it acts as antispermatogenic [25].

\section{Zincum}

Yashada bhasma also known as Zincum comprises mainly the element zinc is a useful micronutrient essential for the smooth functioning of female reproductive system. It was found to be effective in treating leucorrhoea, polycystic ovarian syndrome, as well as to treat endometriosis. Zinc helps to maintain homeostasis and thus plays a very important role in the proper functioning of cells (including their differentiation, growth and division), endocrine and immune system, transcription, synthesis of proteins, RNA and DNA; and DNA replication [26]. Zinc helps in ovulation, fertilization, normal pregnancy, fetal development, and parturition [27-29]. Several animal based studies reported the significance of zinc to prevent impaired synthesis and/or secretion of follicle-stimulating hormone (FSH) and luteinizing hormone (LH), abnormal ovarian development, disruption of the menstrual cycle, prolonged gestation period, abortion, still-births, gross congenital malformation of fetuses, teratogenic effects, delayed and prolonged deliveries with excessive bleeding, difficult parturition, uncoordinated uterine impulses or inefficient uterine contractions, preeclampsia and low birth weights of infants $[30,31]$. Zinc mainly helps to regulate the oocyte maturation through the MOSMAPK (MOS-mitogen activated protein kinase) pathway. Zinc also plays a critical role in fertility as it acts as a cofactor in enzymes of the folate cycle, which are involved in homocysteine recycling to methionine essential during oocyte development.

\section{CONCLUSION:}

Through an extensive literature based evidences provided, it was thus thought worthwhile to combine the above mentioned promising herbs in a single dosage form. The galaxy of phytoconstituents present in the standardized concentrations in the various extracts can be concluded to provide a significant benefit in treating the female 
reproductive disorders. The combination of several herbs shall effectively work via multiple mechanisms to cure polycystic ovarian syndrome, irregular menstruation, different types of menorrhagic conditions as well as treat anovulation, leucorrhea, female infertility and prevent miscarriage. Stree sanjivani as the name suggest thus shall provide a new life to the females, provide the mental as well as physical strength to have a natural and healthy child birth and spread smiles in every family.

\section{REFERENCES:}

[1] Alamoudi, M.O. and Bakrshoom, Y.F. (2021). Vitex agnus castus and Some Female Disorders: A Review. Agricultural Reviews. DOI: 10.18805/ag. R-170.

[2] Bethany Montgomery Hays MD, Tori Hudson ND, in Textbook of Natural Medicine (Fifth Edition), 2020, Vol-2.

[3] Raphael O. Cerqueiral \& Benicio N. Frey2,3 \& Emilie Leclerc 1 \& Elisa Brietzke 1 (2017). Vitex agnus castus for premenstrual syndrome and premenstrual dysphoric disorder: A systematic review. Arch Womens Ment. Health. https://doi.org/10.1007/s00737017-0791-0.

[4] Schellenberg R (2001) Treatment for the premenstrual syndrome with agnus castus fruit extract: prospective, randomised, placebo controlled study. BMJ 322:134-137.
[5] Ma L, Lin S, Chen R, Wang X (2010a) Treatment of moderate to severe premenstrual syndrome with Vitex agnus castus (BNO 1095) in Chinese women. Gynecol Endocrinol 26(8):612-616.

[6] Leah Hechtman MSci (RHHG), BHSc (Nat), ND, in Textbook of Natural Medicine (Fifth Edition), 2020. Vol-2.

[7] Samapika Nandya, Anuradha Mukherjeeb, Devendra Kumar Pandey,Puja Raya, Abhijit Deya. (2020) Indian Sarsaparilla (Hemidesmus indicus): Recent progress in research on ethnobotany, phytochemistry and pharmacology. Journal of Ethnopharmacology. 254. 112609.

[8] Turrini, E., Calcabrini, C., Tacchini, M., Efferth, T., Sacchetti, G., Guerrini, A., Paganetto, G., Catanzaro, E., Greco, G., Fimognari, C., 2018a. In vitro study of the cytotoxic, cytostatic, and antigenotoxic profile of Hemidesmus indicus (L.) R.Br. (Apocynaceae) crude drug extract on $\mathrm{T}$ lymphoblastic cells. Toxins 10, E70.

[9] Turrini, E., Catanzaro, E., Muraro, M.G., Governa, V., Trella, E., Mele, V., Calcabrini, C., Morroni, F., Sita, G., Hrelia, P., Tacchini, M., 2018 b. Hemidesmus indicus induces immunogenic death in human colorectal 
cancer cells. Oncotarget 9, 2444324456.

[10] S. Alok, S.K. Jain, A. Verma, M. Kumar, A. Mahor, M. Sabharwal, Plant profile, phytochemistry and pharmacology of Asparagus racemosus (Shatavari): a review, Asian Pac. J. Trop. Dis. 3 (2013) 242-251.

[11] S.K. Pandey, A. Sahay, R.S. Pandey, Y.B. Tripathi, Effect of Asparagus racemosus rhizome (Shatavari) on mammary gland and genital organs of pregnant rat, Phytother. Res. 19 (2005) 721-724.

[12] K. Sharma, M. Bhatnagar, Asparagus racemosus (Shatavari): a versatile female tonic, IJPBA 2 (2011) 855-863

[13] Ajai K Pandey, Anumegha Gupta, Meenakshi Tiwari, Shilpa Prasad, Ashutosh N. Pandey, Pramod K. Yadav, Alka Sharma, Kankshi Sahu, Syed Asrafuzzaman, Doyil T. Vengayil, Tulsidas G. Shrivastavd, Shail K Chaubeb, Impact of stress on female reproductive health disorders: Possible beneficial effects of shatavari (Asparagus racemosus). Biomedicine and Pharmacotherapy (2018). 103, 4649.

[14] Jetmalani MH, Sabins PB, Gaitonde BB. A study on the pharmacology of various extracts of Shatavari-
Asparagus racemosus (Willd). J Res Ind Med 1967; 2:1-10.

[15] Vandana Baranwal. Asoka: Herbal Boon to Gynecological Problems-An Overview of Current Research Ayurveda Journal of Health VOL. XII, ISSUE 4, FALL 2014

[16] P. Pradhan, L. Joseph, V. Gupta, R. Chulet, H. Arya, R. Verma, A. Bajpai. Saraca asoca (Ashoka): A Review. Journal of Chemical and Pharmaceutical Research, 2009, 1 (1):62-71

[17] Bhaduria Preeti et al., 2012, A Review of Saraca indica plant, IRJP, 3(4): 8084.

[18] MJ Bhandary; KR Chandrasekhar; KMK averiappa. J Ethnopharmacol, $1995,47(3), 149-158$.

[19] Giulia Pastorino, Laura Cornara, Sónia Soares, Francisca Rodrigues, M. Beatriz P.P. Oliveira. Liquorice (Glycyrrhiza glabra): A phytochemical and pharmacological review Phytotherapy Research. 2018;1-17.

[20] Rizzato, G., Scalabrin, E., Radaelli, M., Capodaglio, G., \& Piccolo, O. (2017). A new exploration of licorice metabolome. Food Chemistry, 221, 959-968.

[21] Sharma, G., Kar, S., Palit, S., \& Das, P. K. (2012). 18ß-Glycyrrhetinic acid induces apoptosis through modulation 
of Akt/FOXO3a/Bim pathway in

human breast cancer MCF-7 cells. Journal of Cellular Physiology, 227(5), 1923-1931.

[22]Kim, S. H., \& Park, M. J. (2012). Effects of phytoestrogen on sexual development. Korean Journal of Pediatrics, 55(8), 265-271.

[23] Su Wei Poh, M., Voon Chen Yong, P., Viseswaran, N., \& Chia, Y. Y. (2015). Estrogenicity of glabridin in Ishikawa cells. PLoS One, 10(3), e0121382.

[24] Navneet Kumar, L. G. Abichandani, Vijay Thawani, K. J. Gharpure, M. U. R. Naidu, and G. Venkat Ramana Efficacy of Standardized Extract of Bacopa monnieri (Bacognize ${ }^{\circledR}$ ) on Cognitive Functions of Medical Students: A Six-Week, Randomized Placebo-Controlled Trial. Evid Based Complement Alternat Med. 2016; 2016: 4103423.

[25] A. Singh, S.K. Singh. Evaluation of antifertility potential of Brahmi in male mouse. J Contracep, 79 (2009), pp. $71-79$

[26] Marzenna Nasiadek, Joanna Stragierowicz, Michał Klimczak, and
Anna Kilanowicz. The Role of Zinc in Selected Female Reproductive System Disorders. Nutrients. 2020 Aug; 12(8): 2464.

[27]Ebisch I.M., Thomas C., Peters W., Braat D., Steegers-Theunissen R.P.M. The importance of folate, zinc and antioxidants in the pathogenesis and prevention of subfertility. Hum. Reprod. Update. 2006;13:163-174.

[28] Vallee B.L., Falchuk K.H. The biochemical basis of zinc physiology. Physiol. Rev. 1993;73:79-118.

[29] Bedwal R.S., Bahuguna A. Zinc, copper and selenium in reproduction. Experientia. 1994;50:626-640.

[30] Wang H., Hu Y.-F., Hao J.-H., Chen Y.-H., Su P.-Y., Wang Y., Yu Z., Fu L., Xu Y.-Y., Zhang C., et al. Maternal zinc deficiency during pregnancy elevates the risks of fetal growth restriction: A population-based birth cohort study. Sci. Rep. 2015;5:11262.

[31]Cummings J.E., Kovacic J.P. The ubiquitous role of zinc in health and disease. J. Vet. Emerg. Crit. Care. 2009;19:215-240. 